\title{
SIMULATION OF ELECTROMAGNETIC FIELD DISTRIBUTION IN THE MEASURING CELL FOR DETERMINING THE DIELECTRIC PERMITTIVITY OF MATERIALS AT MICROWAVE FREQUENCIES
}

\author{
NATALIA A. PEVNEVA, ALEKSEI A. KOPSHAI, ALEXANDER L. GURSKII \\ Belarusian State University of Informatics and Radioelectronics (Minsk, Republic of Belarus)
}

Submitted 6 July 2020

(C) Belarusian State University of Informatics and Radioelectronics, 2020

\begin{abstract}
To determine the dielectric permittivity of materials in a wide frequency range with the automation of measurements and the necessary accuracy, measuring cells have been created to ensure the simplicity of the design of the waveguide path. In order to obtain information about the suitability of measuring cells based on irregular SHF waveguides for estimation of dielectric parameters of materials, we simulated the structure of electromagnetic field in the system consisting of two irregular waveguides and waveguide chamber placed between them using a three-dimensional electrodynamic simulation in Ansoft HFSS package environment. The distribution of the electric field was simulated when an empty polyethylene tube, a rod of fluoroplastic and a rod of textolite are placed in the measuring cell. It was demonstrated that high order modes fade out in irregular waveguide and do not affect the precision of obtained results, and significant edge effects were not detected. It allows one to utilize measuring cells based on irregular waveguides together with a scalar or vector network analyzer and using the partial filling of the waveguide method or the modified Nicholson - Ross - Weir method for measurements of dielectric permittivity $\varepsilon$ of materials. The results of modeling the dependence of the amplitude and phase of the reflection coefficient of the textolite and fluoroplastic on the frequency in Ansoft HFSS environment are given. The simulation results are compared with the results obtained experimentally. The frequency dependencies of $\varepsilon$ were obtained experimentally for test materials - textolite and fluoroplastic in the frequency range of 25,95-37,50 GHz. The experimental data are in satisfactory agreement with the results of theoretical calculations and do not go beyond the boundaries specified by the measurement uncertainty.
\end{abstract}

Keywords: electromagnetic field distribution, measuring cell, dielectric permittivity, reflection coefficient.

Conflict of interests. The authors declare no conflict of interests.

For citation. Pevneva N.A., Kopshai A.A., Gurskii A.L. Simulation of electromagnetic field distribution in the measuring cell for determining the dielectric permittivity of materials at microwave frequencies. Doklady BGUIR. 2020; 18(6): 75-80.

\section{Foreword}

Today one can observe the growth in need for precise and reliable contactless methods and instruments to measure dielectric permittivity $\varepsilon$ of materials in a wide range of microwave frequencies. Quite a lot of such methods are known [1], but the means of their implementation are insufficiently developed. As a result of the analysis of existing methods and instruments for measuring the dielectric permittivity $\varepsilon$ of materials, two methods were chosen for constructing measuring instruments: the method of partial filling of the waveguide [2] and the modified Nicholson - Ross - Weir method [3]. They can be used as the basis for measuring the properties $\varepsilon$ of materials under study 
in various aggregate states, provide broadband frequency measurements and automate them, with the necessary measurement accuracy $(\sim 5 \%)$; simplicity of the composite waveguide path design and practical implementation of the measurement technique [4-6].

To create advanced measurement tools based on the methods mentioned above, it is necessary to optimize the geometry of the measurement cells (MC) intended for placement in the waveguide system of the sample under study. This requires accurate information about the spatial structure of the electromagnetic field in a cell, given his distortions caused by the configuration of the waveguide and the introduction of the test sample in the form of a rod in the case of solid materials or dielectric tube which is subsequently filled with sample, if liquid or powdered substances. In particular, to improve the accuracy and reliability of measurements using irregular waveguides, it is necessary to minimize the influence of edge effects and higher-order modes. The purpose of this work is to obtain information about the spatial distribution of the electromagnetic field in the measuring cell for determining the permittivity of materials on the microwave, which would minimize the influence of the above effects.

\section{Routine of calculations and experiment}

In order to obtain information about the structure of the electromagnetic field inside the MC, a system consisting of two irregular waveguides and one waveguide chamber placed between them was simulated using the Ansoft HFSS (High Frequency Structural Simulator) three-dimensional electrodynamic modeling environment [7].

Taking into account the results of numerical modeling, the MC was manufactured as part of a waveguide system used for further connection to a vector circuit analyzer to measure the permittivity of materials. Experimental measurements were carried out on test samples, which were taken as textolite and fluoroplast, since their parameters are well known. For measurements, the method of partial filling of the waveguide was implemented, for which an installation was assembled based on the vector network analyzer "Meter of complex reflection and transmission coefficients R4-MVM-37", a waveguide path in the $25.95-37.50 \mathrm{GHz}$ band and an MC optimized based on the results given below. The installation scheme is described in detail in [5], and the measurement method and accuracy assessment were similar to those given in [5] and [8], respectively.

To obtain the most reliable results, the sample's contribution to the field change must be significant while minimizing interfering factors. To make sure that the assumption is correct that the introduction of the test sample into the MC will cause a significant change in the pattern of electric field propagation, the introduction of a fluoroplast rod and a textolite rod into the MC was modeled.

\section{Results and discussion}

In the HFSS-modeled system, we obtained and analyzed the electric field distribution (Fig. 1).

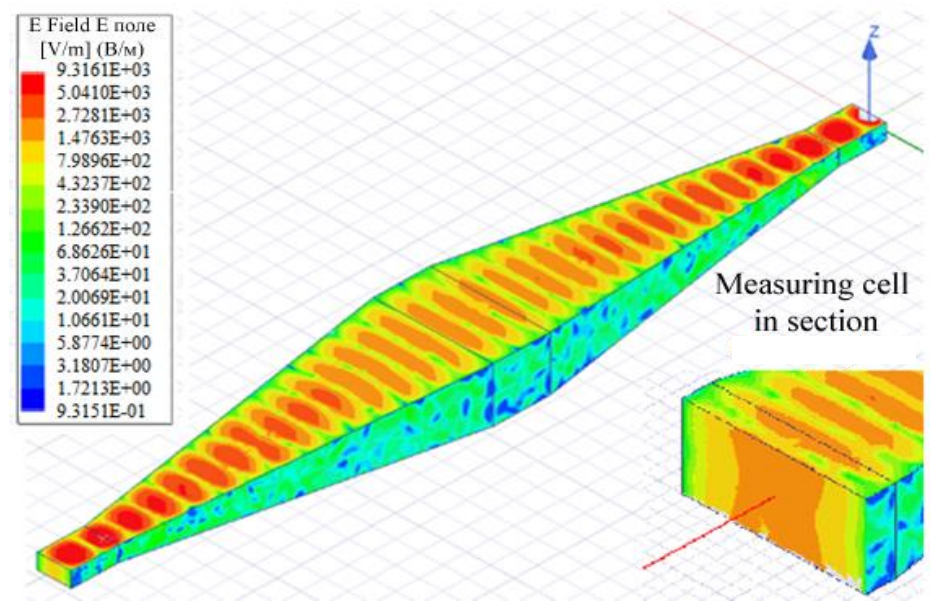

Fig. 1. Scheme of the distribution of the electric field in the simulated measuring cell

To obtain reliable results, it is necessary, in particular, to ensure a sufficient degree of uniformity of the field in the section perpendicular to the waveguide axis at the sample location. 
As can be seen in the box in Fig. 1, this condition is met in a sufficiently large area in the central part of the extended section of the waveguide. If the cross-section dimensions ensure the forming of the necessary field structure, then by gradually increasing the waveguide cross-section, this structure can be preserved, and the dimensions of the measuring cell can be increased. At the point of transition from the waveguide to the horn, higher-order modes appear, but with a sufficiently smooth expansion of the waveguide (a small flare angle of the horn), the intensity of these waves is small (Fig. 2). Fig. 2 represents the upper line (non-zero) for the $\mathrm{H}_{10}$ wave intensity, and the remaining four lines for the $\mathrm{H}_{20}, \mathrm{H}_{30}, \mathrm{H}_{40}$, and $\mathrm{H}_{50}$ waves are near zero, as shown by the simulation results.

Directional properties in an irregular waveguide made in the form of a horn can be estimated approximately using the Kirchhoff-Huygens method [9]. Because the horn basically retains the same field character as the waveguide, they assume that in an aperture there are two field components EY and HX, the amplitude of which does not depend on Y-coordinates, and changes along the X-coordinate according to the cosine law. However, unlike the surface of the open end of the waveguide, the aperture of the horn cannot be excited in phase, since a cylindrical (in sectorial horns) or close to spherical (in pyramidal horns) wave propagates in the horn.

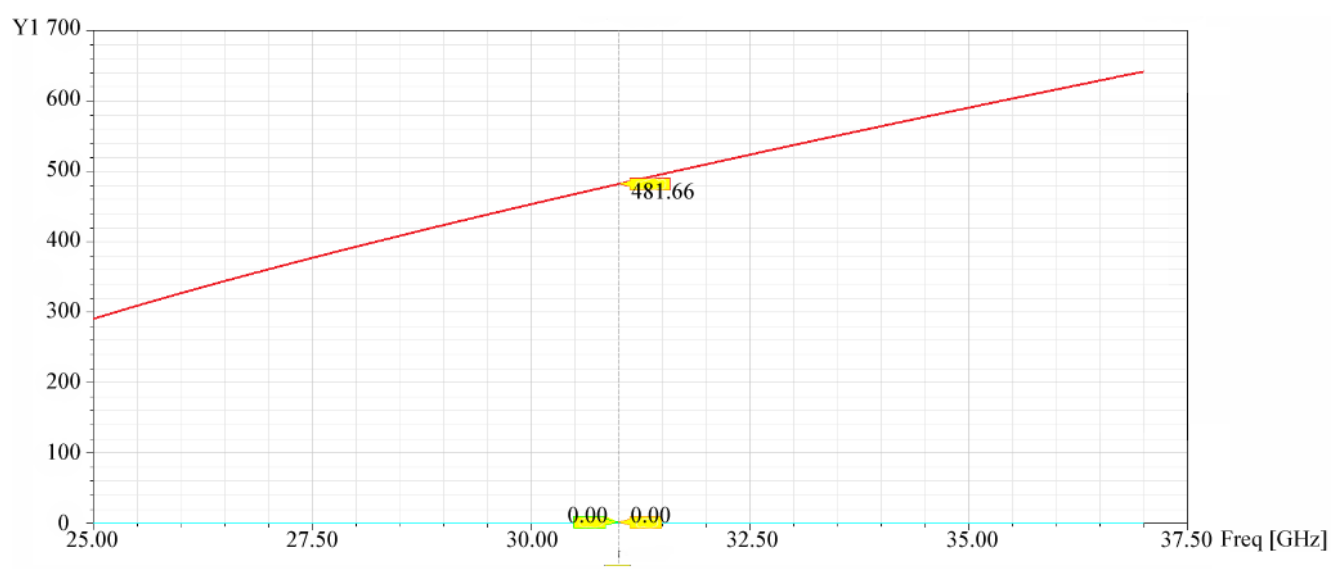

Fig. 2. Frequency dependence of the wave intensities $\mathrm{H}_{10}, \mathrm{H}_{20}, \mathrm{H}_{30}, \mathrm{H}_{40}, \mathrm{H}_{50}$

Three cases were modeled for materials with known parameters in order to consider the behavior of the electric field in the MC when a dielectric rod is inserted into it in the HFSS environment. Fig. 3 shows the distribution of the electric field when an empty polyethylene tube is placed in the MC, Fig. 4 demonstrates a rod made of fluoroplast, and Fig. 5 - a rod made of textolite. Fig. 3-5 depict the propagation of the $\mathrm{H}_{10}$ wave on the left and the $\mathrm{H}_{20}$ wave on the right. Computer simulation determined that higher-order modes are damped in an irregular waveguide and do not affect the accuracy of the results obtained, as well as no significant edge effects were found.
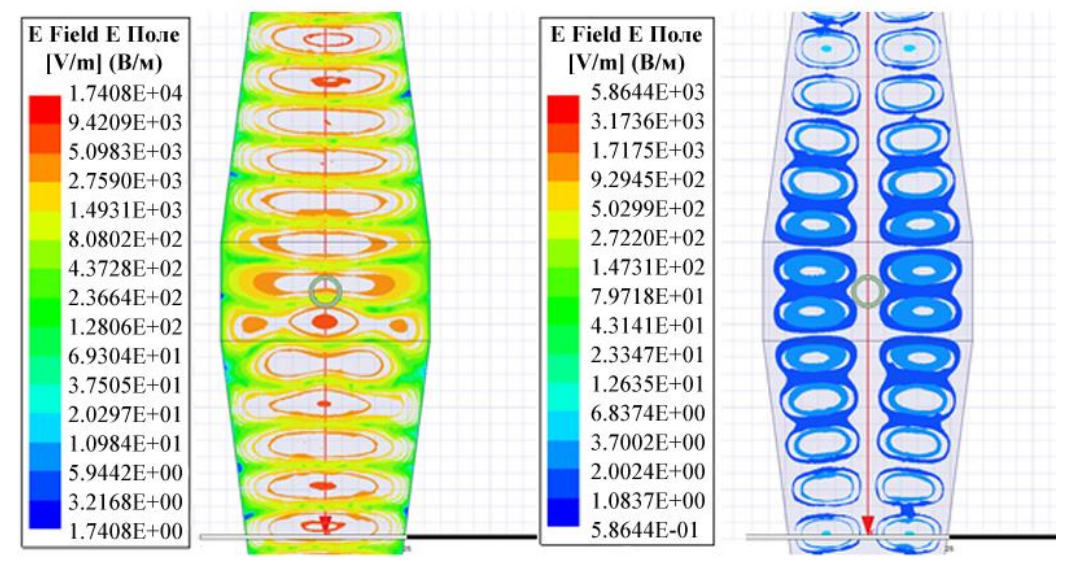

Fig. 3. The distribution of the electric field when an empty polyethylene tube is placed in the measuring cell (on the left wave propagation $\mathrm{H}_{10}$, on the right $-\mathrm{H}_{20}$ ) 


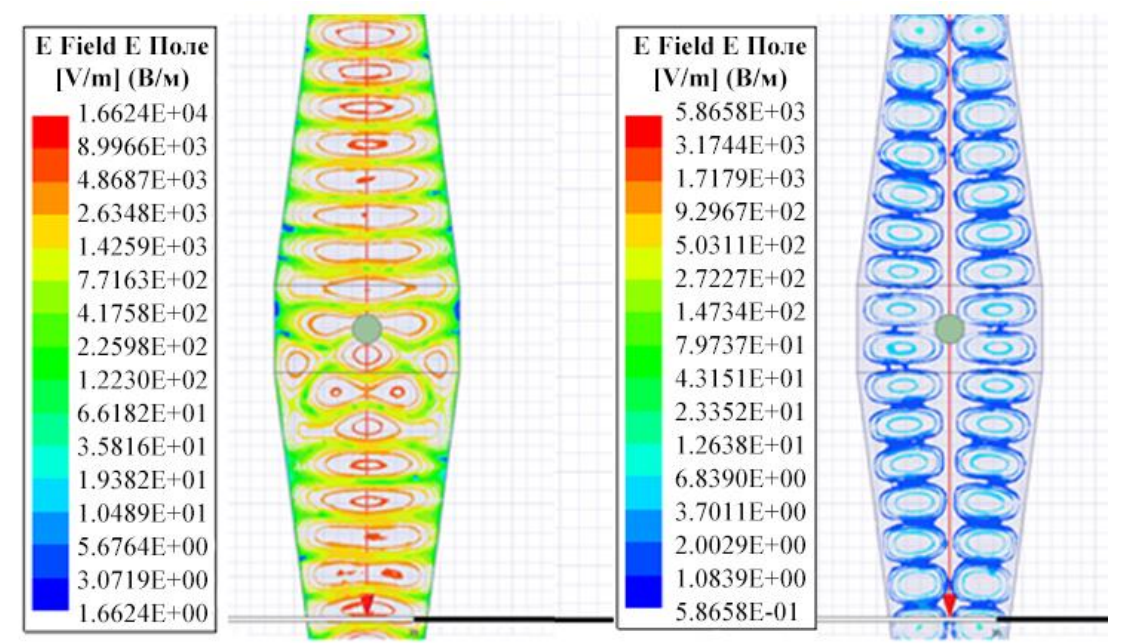

Fig. 4. The distribution of the electric field when the rod of fluoroplastic is placed in the measuring cell (on the left wave propagation $\mathrm{H}_{10}$, on the right $-\mathrm{H}_{20}$ )

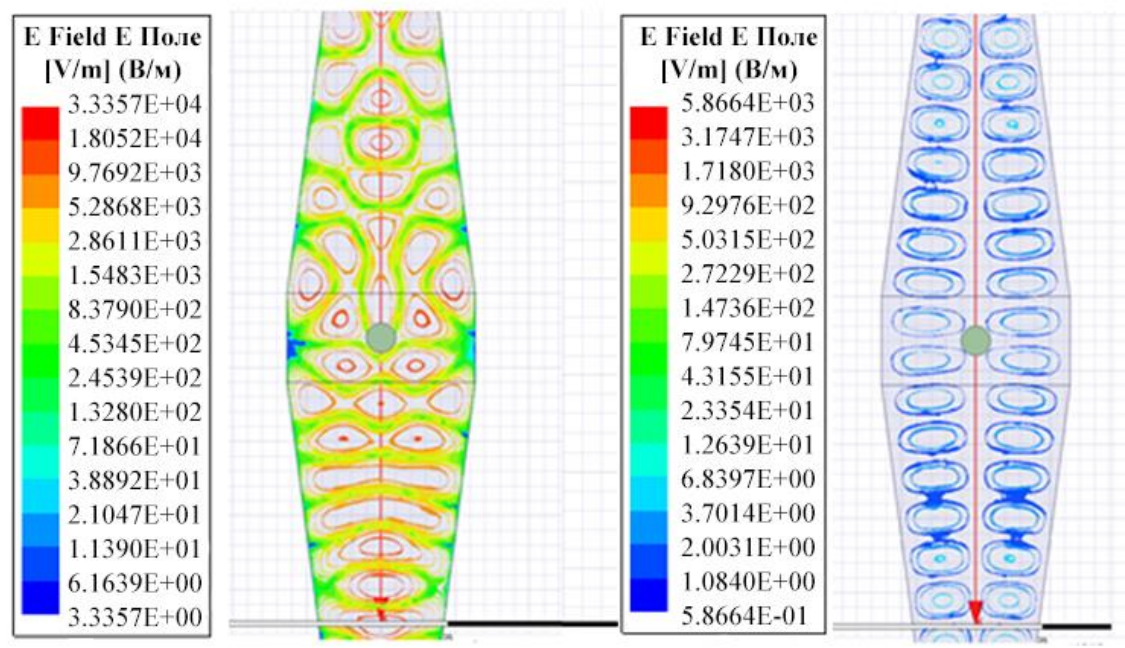

Fig. 5. The distribution of the electric field when the rod of textolite is placed in the measuring cell (on the left wave propagation $\mathrm{H}_{10}$, on the right $-\mathrm{H}_{20}$ )

Since the method for measuring the permittivity is based on finding the S-parameters, HFSS modeled the propagation of the reflected wave in the measuring cell and obtained a graph of the dependence of $S_{11}$ on the frequency in the frequency range $25.95-37.50 \mathrm{GHz}$ (Fig. 6). Sharp dips in the dependence that occur at a number of frequencies can be eliminated by calibrating the measurement path during measurements.

Based on the results obtained, we optimized the geometric dimensions and shape of a MC made for measuring the dielectric parameters of materials in the frequency range $25.95-37.50 \mathrm{GHz}$ in conjunction with a scalar or vector circuit analyzer. The MC is assembled from two horn antennas and a waveguide chamber placed between them, in which an insert with a hole for rod-shaped samples is placed.

The optimized measurement cell was used in conjunction with a vector circuit analyzer to study the dependence of the amplitude $\left(S_{11}\right)$ and phase $\left(\varphi_{11}\right)$ of the reflection coefficient of a number of materials on the frequency $f$. As an example, Fig. 7 shows the results for fluoroplast $(a, b, d)$ and textolite $(b, d, e)$ in the frequency range 25.95-37.50 GHz. In particular, the experimental (solid lines) and HFSS-modeled (dotted lines) dependences of the amplitude $(a, b)$ and phase $(c, d)$ of the reflection coefficient on frequency are shown, as well as the obtained dependences of $\varepsilon$ on frequency $(d, e)$. 


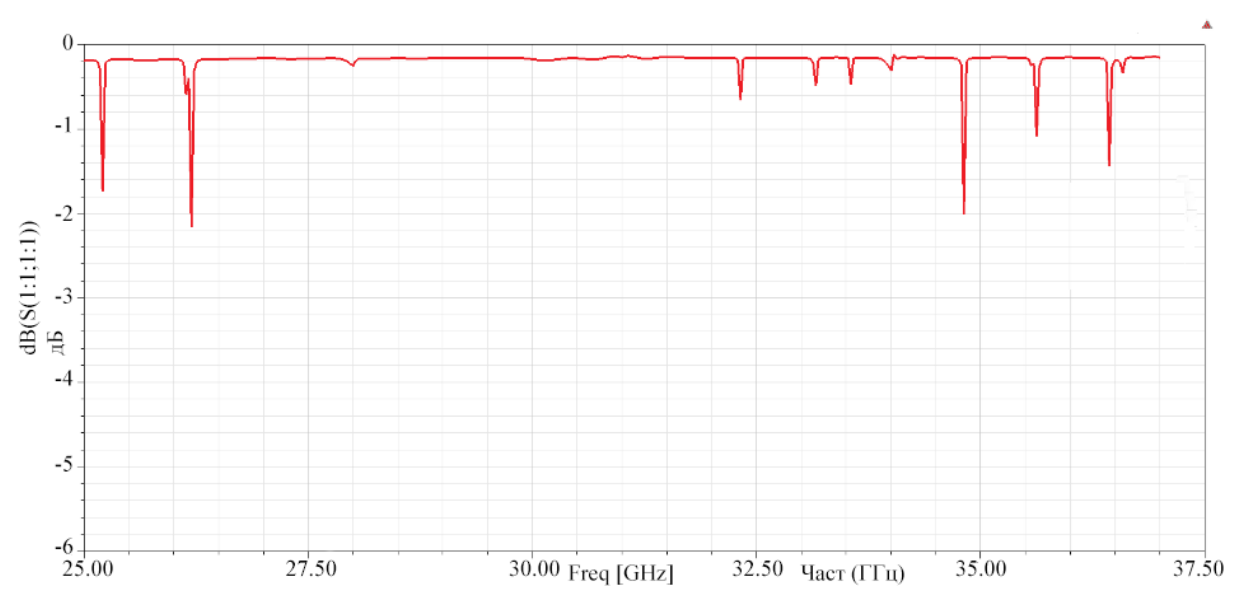

Fig. 6. Dependence of $S_{11}$ on frequency in the frequency range $25.95-37.50 \mathrm{GHz}$
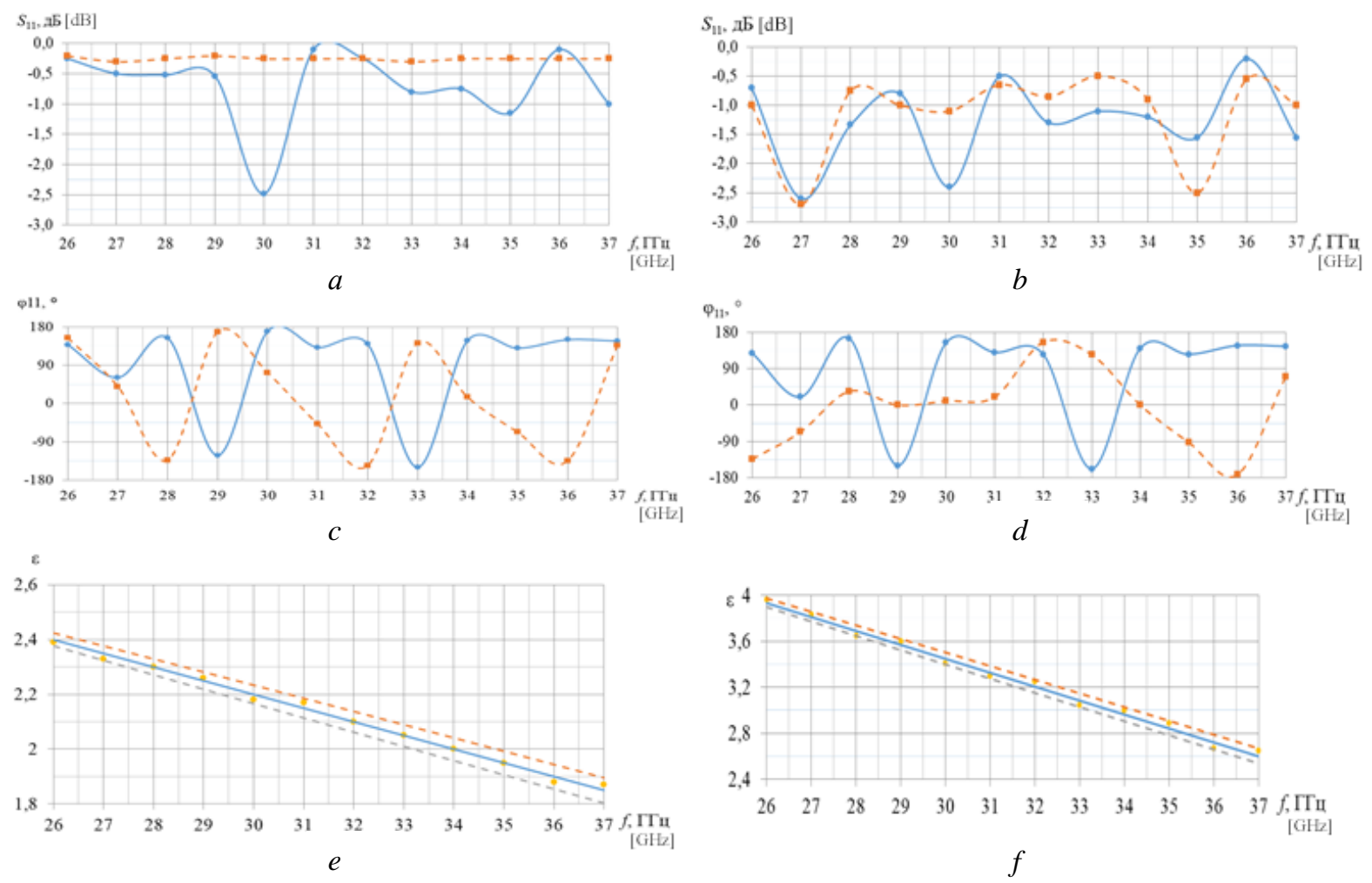

Fig. 7. Experimental (solid lines) and simulated in HFSS (dashed lines) dependences of the amplitude $(a, b)$ and phase $(c, d)$ of the reflection coefficient on frequency, as well as the obtained dependences of $\varepsilon$

on frequency $(e, f)$ for fluoroplastic $(a, c, e)$ and textolite $(b, d, f)$ in the frequency range $25.95-37.50 \mathrm{GHz}$ (in Figures $(e)$ and $(f)$ the dashed lines show the boundaries of the region of uncertainty of the obtained results)

The results of numerical modeling of the amplitude and phase of the reflection coefficient were used as input parameters in the mathematical model for calculating the permittivity and it was found that the permittivity calculated theoretically does not exceed the limits set by the measurement uncertainty.

\section{Conclusion}

As a result of numerical modeling and analysis of electric field distribution in the measurement cell of microwave range, consisting of a pair of irregular waveguides with a waveguide chamber in-between, in the absence and presence of the rod-shaped samples of a certain size, we have established that in this system there is a significant impact of the presence of samples on the spatial structure of the field, while the influence of edge effects and higher-order modes is reduced to a negligibly small level in comparison with permissible measurement uncertainty. 
Based on the obtained data, measurement cells are developed that are used in the implementation of the method of partial filling of the waveguide and the modified Nicholson - Ross Weir method, which are the basis for measuring the properties of various materials in liquid and solid states. This ensures broadband frequency measurements, their automation, and the necessary accuracy $(\sim 5 \%)$ with a simple design of the composite waveguide path. The experimental data obtained for the test materials are in agreement with the data of theoretical calculations and do not exceed the limits set by the uncertainty of the measurement results.

\section{References}

1. Brandt A.A. [Investigation of dielectrics at microwave frequencies]. Moscow: FIZMATLIT; 1963. (In Russ.)

2. Radin Yu.P. [On a method for measuring the dielectric constant in the centimeter range]. Izvestia VUZov. Radiofizika. 1958;1(5-6):177-179. (In Russ.)

3. Luukkonen O., Maslovski S.I., Tretyakov S.A. A stepwise Nicolson-Ross-Weir-based material parameter extraction method. IEEE Antennas and Wireless Propagation Letters. 2011;10:1295-1298.

4. Pevneva N.A., GusinskyA. V., GurskiiA. L. [Microwave method for determining the dielectric properties of liquids]. Doklady BGUIR = Doklady BGUIR. 2012;5(67):46-50. DOI: 10.35596 (In Russ.)

5. Pevneva N. A., Gurskii A. L., Kostrikin A. M. [Using the method of a cylindrical rod and a vector network analyzer to determine the dielectric constant of materials in the microwave range]. Doklady BGUIR= Doklady BGUIR. 2019;1(119):56-61. DOI: 10.35596 (In Russ.)

6. Pevneva N.A., Gurskii A.L., Kostrikin A.M. [The free space method using a vector network analyzer for determining the permittivity of materials at microwave frequencies]. Doklady BGUIR = Doklady BGUIR. 2019;4(122):32-39. DOI: 10.35596 (In Russ.)

7. Bankov S.E., Kurushin A.A. [Calculation of antennas and microwave structures using HFSS Ansoft]. Moscow: ZAO "NPP RODNIK”; 2009. (In Russ.)

8. Pevneva N.A., Gurskii A.L., Kostrikin A.M. [Estimation of the uncertainty of measurements of the dielectric permittivity of materials by the method of a cylindrical rod on a microwave]. Metrologia i priborostroenie. 2019;2:30-34. (In Russ.)

9. Frolov O.P. [Antennas and feeder paths for radio relay communication lines]. Moscow: Radio i svyaz'; 2001. (In Russ.)

\section{Authors' contribution}

Pevneva N.A. - research concept and design, data analysis and interpretation, article preparation for publication, treatment of the obtained experimental results and preparation of the article for publication.

Kopshai A.A. - simulation of the distribution of the electric field and interpretation of the results.

Gurskii A.L. - editing and final approval of the manuscript for publication, its critical review in terms of significant intellectual content.

\section{Information about the author}

Pevneva N.A., Researcher of the Center 1.9 of R\&D Department of Belarusian State University of Informatics and Radioelectronics.

Kopshai A.A., Researcher of the Center 1.9 of R\&D Department of Belarusian State University of Informatics and Radioelectronics.

Gurskii A.L, D.Sci., Professor of the Information Protection Department of Belarusian State University of Informatics and Radioelectronics.

\section{Address for correspondence}

220013, Republic of Belarus,

Minsk, P. Brovka str., 6,

Belarusian State University

of Informatics and Radioelectronics

tel. +375-17-293-20-92;

e-mail: saphir-n@mail.ru

Pevneva Natalia Alekseevna 